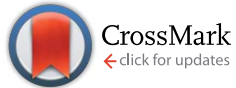

Cite this: RSC Adv., 2017, 7, 14902

Received 5th February 2017

Accepted 17th February 2017

DOI: 10.1039/c7ra01470h

rsc.li/rsc-advances

\section{Enhancement of fluorescent resonant energy transfer and the antenna effect in DNA structures with multiple fluorescent dyes $\uparrow$}

\begin{abstract}
Taeseok Oh, ${ }^{\text {ab }}$ Sejung Kim, ${ }^{\mathrm{b}}$ Jae-Young Choi, ${ }^{\mathrm{b}}$ Haeun Chang ${ }^{\mathrm{b}}$ and Michael J. Heller*b
This study examines the use of surfactants and metal cations for the enhancement of long range fluorescent resonant energy transfer (FRET) and the antenna effect in double-stranded (ds) DNA structures formed by hybridization of 21 mer oligonucleotides with three fluorescent TAMRA donor dyes and complementary 21mer oligonucleotides with one fluorescent Texas Red acceptor dye. In FRET ds-DNA structures, hydrophobic interactions between the fluorescent dyes in close proximity produces dimerization and quenching which reduces fluorescent emissions. For the neutral surfactant Triton X-100, dimerization and emission quenching in the FRET ds-DNA structures remain unaffected. The cationic surfactant CTAB $(>100 \mu \mathrm{M})$, which neutralizes the negatively charged ds-DNA backbone reduces TAMRA dye dimerization and emission quenching, and improves the Texas Red quantum yield, FRET efficiency and the antenna effect. While the negatively charged SDS surfactant does not reduce dimerization and emission quenching, addition of sodium cations $\left(\mathrm{Na}^{+}\right)$and magnesium cations $\left(\mathrm{Mg}^{2+}\right)$ lead to a significant reduction in dimerization and emission quenching, and produce higher FRET efficiency and enhanced antenna effect. This study provides a viable strategy for using combinations of surfactants and cations to reduce fluorescent dye and other quenching mechanisms and improve the overall long distance FRET efficiency and the antenna effect in ds-DNA structures.
\end{abstract}

\section{Introduction}

Fluorescence resonance energy transfer (FRET) is a widely used technique to study the structure and dynamics of biomolecules..$^{1,2}$ DNA structures with fluorescent donor and acceptor dyes have been used as molecular sensors, where the ratio of donor and acceptor fluorescent emissions changes according to the hybridization status or as a result of an enzymatic reaction., ${ }^{3,4}$ Additionally, it should be possible to design DNA structures with a precisely spaced array of donor and acceptor fluorophores to collect and transfer photonic energy over longer ranges/distances via coupled FRET events., ${ }^{5,6}$ Such DNA structures could act as photonic antennas and wires, which would have applications in molecular diagnostics and many other areas. ${ }^{7-9}$ Because FRET efficiency among other parameters is determined by a $1 / R^{6}$ distance dependency relationship, ${ }^{10}$ under ideal (theoretical) conditions the optimal distance for positioning donor to donor and donor to acceptor fluorescent groups on a DNA strand is from about $0.34 \mathrm{~nm}$ to $1.4 \mathrm{~nm}$ or one to four base spacing. At

\footnotetext{
${ }^{a}$ Materials Science and Engineering Program, University of California, 9500 Gilman Dr., La Jolla, San Diego, CA 92093, USA

${ }^{b}$ Department of NanoEngineering, University of California, 9500 Gilman Dr., La Jolla, San Diego, CA 92093, USA. E-mail: mheller@ucsd.edu

$\dagger$ Electronic supplementary information (ESI) available. See DOI: $10.1039 / \mathrm{c} 7 \mathrm{ra} 01470 \mathrm{~h}$
}

distances from $1.7 \mathrm{~nm}$ to $2.4 \mathrm{~nm}$ or five to seven base spacing, the FRET efficiency is intermediate to low, while beyond $2.8 \mathrm{~nm}$ or eight base spacing, the FRET efficiency drops off dramatically. Thus, in DNA structures with multiple fluorescent donor groups designed for long range FRET, the donor to donor dye resonant energy transfer efficiency must be intermediate to high in order for the energy that was collected by the more distant donors (beyond $1.7 \mathrm{~nm}$ ) to reach the fluorescent acceptor dye. However, when multiple fluorophores are in a close proximity and can contact each other, the resulting hydrophobic stacking interactions lead to the formation of donor/donor and donor/acceptor dimers. ${ }^{11,12}$ Such contact or dimer formation between the dyes can reduce the dyes quantum yield and fluorescent emission, significantly reducing overall FRET efficiencies in DNA structures designed for long range transfer and antenna properties. ${ }^{13,14}$ Quenching of fluorophores on the DNA structures can also occur by other mechanisms which include contact with water or other polar molecules, as well as by contact with DNA bases where electron-transfer from the base to the excited singlet state of a dye can sometime quench the fluorescence emission. ${ }^{15-19}$ Such DNA base quenching is mostly due to guanine, which has the lowest oxidation potential among the four DNA bases. ${ }^{20,21}$ As was reported in earlier studies, ${ }^{22,23}$ the interaction and association of fluorescent dye conjugated ds-DNA structures with certain surfactant micelles and metal ions were found to significantly reduce dimerization and emission quenching and improve short 
range FRET efficiencies, where the donor to acceptor dye distance was less than $1.4 \mathrm{~nm}$ or four base pairs. In this study, we now investigate the effect of surfactants and metal cations on reducing quenching and enhancing the long range FRET efficiency and antenna effects in 21mer ds-DNA structures with different arrangements of three TAMRA donor dyes and a single Texas Red acceptor dye. In these DNA structures, efficient donor to donor FRET is required as the distance between the donor dyes to the acceptor dye becomes greater than $1.7 \mathrm{~nm}$ or five base spacing and beyond the optimal FRET distance.

\section{Method and material}

Both dye-conjugated 21mer single-stranded (ss) oligonucleotides and 21mer un-conjugated single-stranded (ss) oligonucleotides were supplied by Trilink Inc, San Diego, CA. For the donor 21mer ss-oligonucleotides, three fluorescent TAMRA dyes (carboxytetramethyl-rhodamine) were attached to the thymine bases via C6-linkers (six methylene groups) with a 3 base spacing (5'-TGT GTG (dT-C6-NH-5-TAMRA) GTG (dT-C6-NH-5TAMRA) TTT (dT-C6-NH-5-TAMRA) TGG TTT-3') and a 7 base spacing (5'-TG (dT-C6-NH-5-TAMRA) GTG TGT G (dT-C6-NH-5TAMRA) T TTT TGG (dT-C6-NH-5-TAMRA) TT-3'). For the acceptor 21mer ss-oligonucleotides, a single fluorescent Texas Red (sulforodamine) dye was attached via C6-linkers (six methylene groups) to the 10th adenine base from the $5^{\prime}$-terminal position (5'-AAA CCA AAA A (dA-C6-NH-Texas Red-X) C ACA CAC ACA $\left.-3^{\prime}\right)$, and to the 3 rd adenine base located from the $5^{\prime}$-terminal position (5'-AA (dA-C6-NH-Texas Red-X) CCA AAA AAC ACA CAC ACA-3'). A control complementary 21mer oligonucleotide without a Texas Red dye was also synthesized. The concentrations of the ss-DNA oligonucleotide solutions were determined by UV-Vis absorbance measurements. For preparing the hybridized double-stranded (ds) DNA structures, $1 \mu \mathrm{M}$ of the three TAMRA conjugated DNA (donor sequence I and II) was mixed with $1 \mu \mathrm{M}$ of the Texas Red conjugated complementary strand (acceptor sequence III and IV) or with the blank complementary strand (no acceptor sequence V) in $0.5 \times \mathrm{PBS}$, then heated to $60{ }^{\circ} \mathrm{C}$ and cooled down slowly to room temperature $20{ }^{\circ} \mathrm{C}$ for 2 hours. Fluorescent emission and absorbance spectra of the hybridized ds-DNA FRET structures were obtained using a multi-mode Microplate reader (Infinite M200 Pro, Tecan Inc). The standard excitation and emission values for the TAMRA donor dyes are (Ex. $\max 555 \mathrm{~nm}$ ) and (Em. max $580 \mathrm{~nm}$ ), and for the Texas Red dye are (Ex. max $595 \mathrm{~nm}$ ) and (Em. max $615 \mathrm{~nm}$ ). However, in this study, the excitation and emission maximum values can vary in several nanometers due to the effects of the DNA structure and local environment on the conjugated donor and acceptor dyes. The FRET efficiency of the donor-acceptor DNA hybrids was measured experimentally in several ways. Basically, quantification methods for FRET efficiency require monitoring the change of emission intensity or quantum yield of the acceptor fluorophore with respect to presence and absence of the donor fluorophore. ${ }^{8}$ However, in the multi-donor and single acceptor DNA systems, where the emission intensity of the donors is highly influenced (quenched) by dimerization, the lower FRET efficiencies are more difficult to quantify exactly. However, they do set a reliable lower limit for the other measurements. The long distance FRET transfer and antenna effect measurements are an effective way to characterize the overall light-harvesting or collection capability of the multi-donor single acceptor DNA structures. In order to quantify the FRET performance for the three TAMRA donors and one Texas Red acceptor ds-DNA structures under each of the environmental conditions (with and without surfactants and metal ions), the antenna effect value was determined by measuring the intensity ratio of Texas Red acceptor emission when multiple TAMRA donors are excited at $555 \mathrm{~nm}$, to the Texas Red acceptor emission when the acceptor alone is excited at $595 \mathrm{~nm}$, as shown in the equation below.

$$
\text { Antenna effect }=\frac{\text { acceptor emission }_{\text {multiple donors excited at } 555 \mathrm{~nm}}}{\text { acceptor emission }}
$$

In this study, all measurements were repeated three times for more reliable results. Generally, the distance between donor to donor and donor to acceptor groups is referred as the Förster distance. Among other parameters, FRET efficiency has a $1 / R^{6}$ distance dependency with the Förster distance. Under ideal or theoretical conditions, the FRET efficiencies for a single fluorescent donor and single fluorescent acceptor conjugated to a ds-DNA structure would be high (100\% to $80 \%)$ for Förster distances from about $0.34 \mathrm{~nm}$ to $1.4 \mathrm{~nm}$ (1 to 4 base spacing); intermediate (70\% to $40 \%$ ) for Förster distances from $1.7 \mathrm{~nm}$ to $2.4 \mathrm{~nm}$ ( 5 to 7 base spacing); and low to no FRET (30\% to $0 \%$ ) for distances greater than $2.8 \mathrm{~nm}$ or 8 base spacing, where the FRET efficiency drops off rapidly to $0 \%$ because of the $1 / R^{6}$ distance dependency. For a single donor and single acceptor system at the closest Förster distance and under ideal conditions, the maximum FRET efficiency cannot be greater than 100\%, nor can it have a value greater than 1 for the emission acceptordonor excited/emission acceptor-acceptor excited ratio. However, in cases where there are multiple donors including donors beyond the Förster distance that have efficient donor to donor FRET that produces an antenna effect, then the overall FRET efficiency measured for a system with multiple donors and a single acceptor group can be greater than $100 \%$, and it can have an antenna effect value greater than 1 for the acceptor emission, as determined by the antenna effect equation. By way of an example, an antenna effect value of 1.5 would mean that the ds-DNA structure with multiple TAMRA donors excited at $555 \mathrm{~nm}$ produced a $150 \%$ increase in the Texas Red acceptor emission at $610 \mathrm{~nm}$, compared to the maximum emission value at $610 \mathrm{~nm}$ for the single Texas Red acceptor when excited at $595 \mathrm{~nm}$.

\section{Result and discussion}

In order for the better interpretation of the experimental results, Table 1 shows the actual base sequences and positions of the fluorescent TAMRA donor dyes and the fluorescent Texas Red acceptor dye in the ss-DNA and ds-DNA structures, while Fig. 1 shows the schematic representations of ss-DNA sequences and the hybridized FRET ds-DNA structures. The control A and B three TAMRA dye ss-DNA sequences and control $\mathrm{C}$ and D 
Table 1 DNA sequences and the positions of donor and acceptor dyes

\begin{tabular}{ll}
\hline DNA name & Sequence \\
\hline Donor sequence I & $5^{\prime}$-TGT GTG (dT-C6-NH-5-TAMRA) GTG (dT-C6-NH-5-TAMRA) TTT (dT- \\
& C6-NH-5-TAMRA) TGG TTT-3' \\
Donor sequence II & $5^{\prime}$-TG (dT-C6-NH-5-TAMRA) GTG TGT G (dT-C6-NH-5-TAMRA) T TTT \\
& TGG (dT-C6-NH-5-TAMRA) TT-3' \\
Acceptor sequence III & $5^{\prime}$-AAA CCA AAA A (dA-C6-NH-Texas Red-X) C ACA CAC ACA-3' \\
Acceptor sequence IV & $5^{\prime}$-AA (dA-C6-NH-Texas Red-X) CCA AAA AAC ACA CAC ACA-3' \\
No acceptor sequence V & $5^{\prime}$-AAA CCA AAA AAC ACA CAC ACA-3'
\end{tabular}

hybridized three TAMRA dye ds-DNA structures without the presence of the Texas Red dye in the complementary strand are shown in Fig. 1(a), and the FRET E, F, G \& H three TAMRA donor dye hybridized ds-DNA structures with the Texas Red acceptor dye present in the complementary sequence are shown in Fig. 1(b). In this study, the results for the control ss-DNA, control ds-DNA hybrid structures, and FRET ds-DNA hybrid structures without surfactants or metal ions are designated as 'pristine'. With regard to the FRET ds-DNA hybrid structures E, F, $\mathrm{G}$ and $\mathrm{H}$, the ds-DNA hybrid structure ' $\mathrm{G}$ ' represents the best test case for demonstrating the long range FRET transfer and the antenna effect.

\subsection{Three TAMRA dye conjugated DNA structures without Texas Red acceptor}

Before carrying out FRET experiments on the ds-DNA structures with multiple TAMRA donor groups and a single Texas Red

(a)

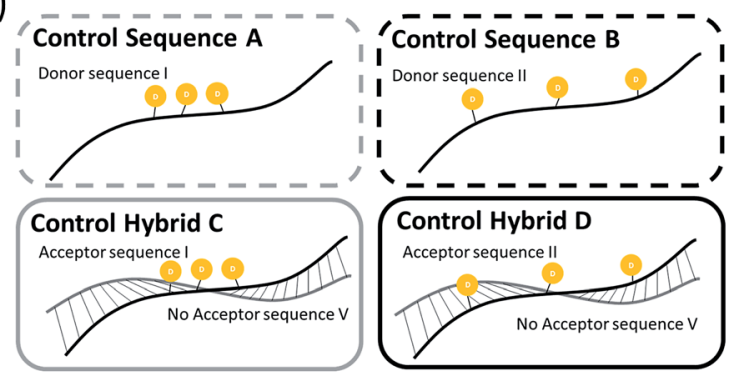

(b)
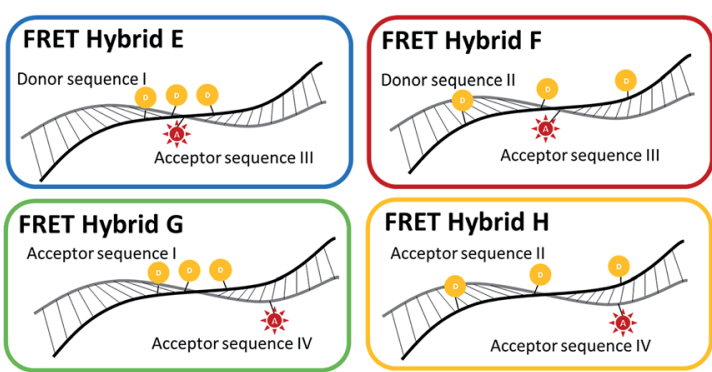

Fig. 1 Schematics representation for: (a) the three fluorescent TAMRA donor dyes on ss-DNA sequences and in ds-DNA hybrid structures without the presence of the fluorescent Texas Red acceptor dye; and (b) the three fluorescent TAMRA donors in FRET ds-DNA hybrid structures with the fluorescent Texas Red acceptor dye present in the complementary sequence. acceptor group in the complementary oligonucleotide strand, the fluorescence and UV absorbance properties of the three TAMRA donor ss-DNA (control sequence A and B) and the dsDNA (control hybrid $\mathrm{C}$ and $\mathrm{D}$ ) with the 3 base and 7 base spacing and no Texas Red acceptor present in the complementary DNA strand, were tested without surfactants or metal ions. As shown in Fig. 2(a), without the addition of surfactants and metal ions, lower fluorescent emission intensities were observed for the TAMRA dyes in the ss-DNA sequences A, B and the hybridized ds-DNA sequence $\mathrm{C}$ indicating significant quenching of the dyes, while a higher emission intensity was observed for the hybridized ds-DNA sequence D indicating much less quenching occurred in this structure. This reduced quenching in the ds-DNA sequence is D may be attributed to the 7 base spacing of the TAMRA dyes together with the more rigid double-stranded hybrid structure not allowing any contact to occur between the dyes. Additionally, the absorbance spectrum intensities for the TAMRA dyes in the ss-DNA sequence A, B and the ds-DNA structure $\mathrm{C}$, increased at $524 \mathrm{~nm}$ and decreased at $556 \mathrm{~nm}$ when comparing to absorbance spectrum of TAMRA dyes in ds-DNA D (Fig. 2(b) and S1 $\dagger$ ). The changes in emission and absorbance spectra are mostly attributed to the static quenching by dimerization of TAMRA fluorophores. As it has been reported in the previous studies, ${ }^{22,23}$ contact of fluorescent dyes in aqueous solutions often leads to dimerization, especially if the local concentration of hydrophobic fluorophores is high or they are in close proximity, for example, when they are conjugated in a close proximity on a DNA molecule. It is also well-known that formation of homo- and heterodimers can cause distinct changes in the absorption spectrum due to coupling of the excited-state energy levels. In terms of H-type aggregates, absorption is allowed only to the upper area of the excited-state, absorption is blue-shifted and the fluorescence emission intensity is diminished. ${ }^{\mathbf{2 4 2 5}}$ Thus, changes in the absorbance spectrum confirm that the emission quenching is induced by the dimerization of the fluorophore dyes, where the absorptions at $556 \mathrm{~nm} 524 \mathrm{~nm}$ correspond specifically to the TAMRA monomer and dimer, respectively. As seen in Fig. 2(b), lower $556 \mathrm{~nm}$ monomer to $524 \mathrm{~nm}$ dimer ratios were observed for the TAMRA dyes in the ss-DNA sequences A and B and the hybridized ds-DNA sequence $\mathrm{C}$, indicating significant dimerization of the dyes, while a higher ratio was observed for the hybridized ds-DNA sequence D indicating much less dimerization. Significant dimerization would be expected in ss-DNA 

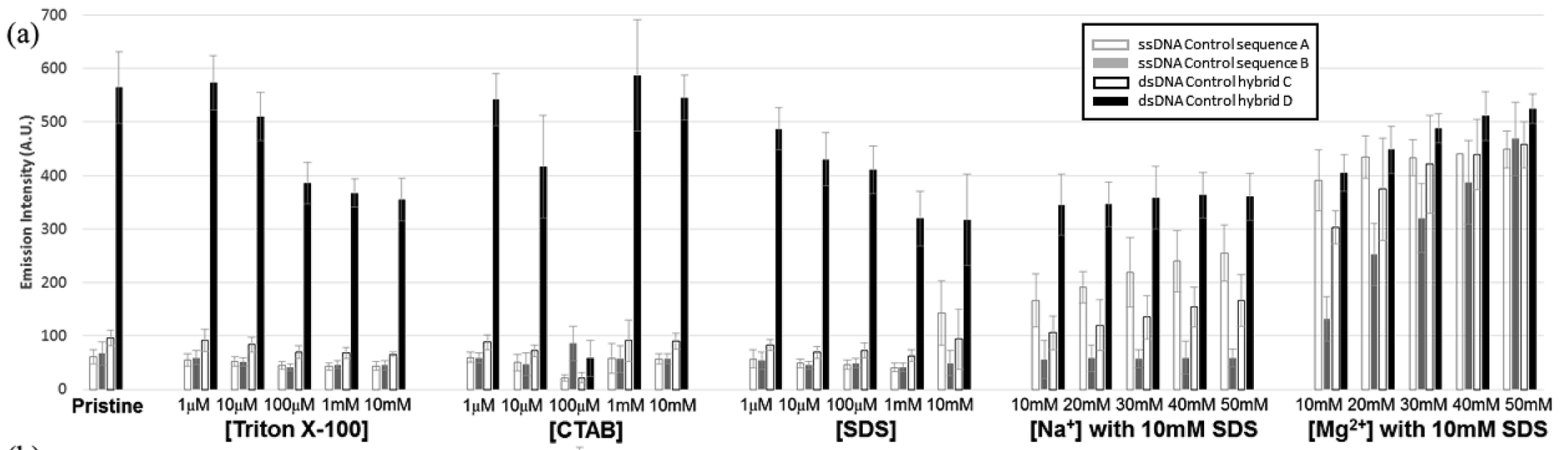

(b)
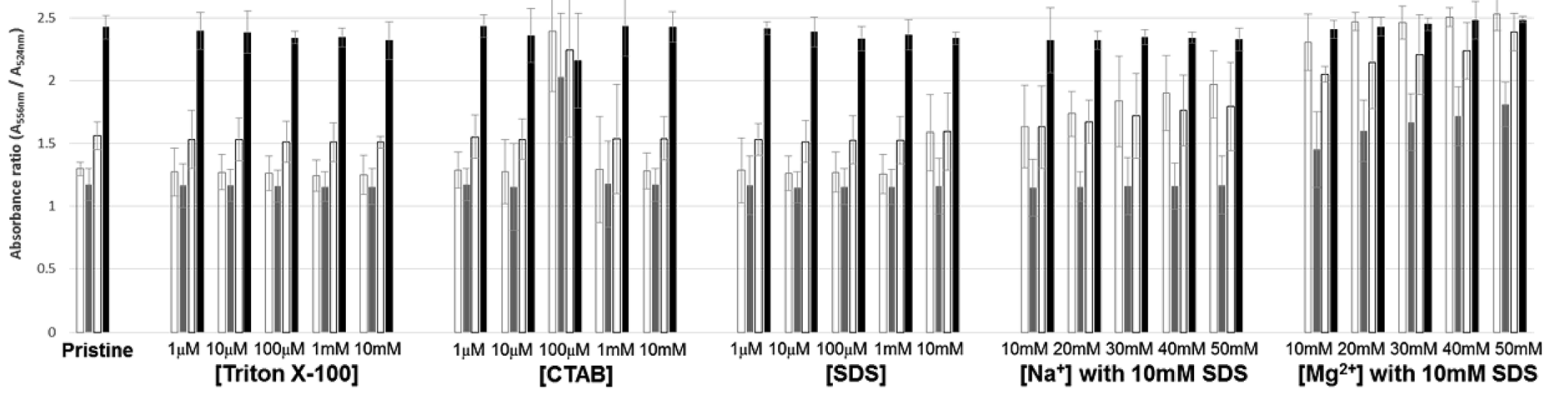

Fig. 2 Fluorescent and UV absorption spectral analysis results for the three fluorescent TAMRA donor dye ss-DNA sequences (A and B) and dsDNA hybrid structures ( $C$ and $D$ ) without the Texas Red acceptor dye present: (a) maximum fluorescence emission intensities for the three fluorescent TAMRA donor dye ss-DNA structures with and without surfactants and (b) the monomer absorbance spectra ratios $\left(A_{556} \mathrm{~nm} / A_{524} \mathrm{~nm}\right)$ for the control sequences A (blank gray) and B (blank black), and the control ds-DNA hybrids C (filled gray) and D (filled black) by Fig. S1. $\dagger$

sequence $\mathrm{A}$ and ds-DNA $\mathrm{C}$ with the shorter 3 base spacing of the TAMRA groups, as well as in the ss-DNA sequence $\mathrm{C}$ which even though it has 7 base spacing of the TAMRA groups, the single stranded DNA structure is flexible enough to allow the TAMRA groups to make contact. However, the ds-DNA sequence D with 7 base spacing is rigid enough to prevent any TAMRA dye contact from occurring. When Triton X-100 was added to the solution, the emission quenching and dimerization were still observed for the three TAMRA dyes in ss-DNA sequences A and $\mathrm{B}$, and the ds-DNA sequence C (Fig. 2). Because Triton X-100 is un-charged (neutral), the surfactant has little interaction with negative charged DNA backbone and does not appear to affect the dimerization of the TAMRA dyes. However, the emission intensities of the TAMRA dyes in the ds-DNA structure D decreased as the Triton X-100 concentration increased due to the less polar environment. ${ }^{26}$ It is well known that rhodamine type dyes such as TAMRA are highly fluorescent in polar solvents and the high quantum yield is maintained by restricting the rotation of the phenyl rings in rhodamine dyes by a bridging oxygen atom. ${ }^{27}$ Thus, as the Triton X-100 cause the aqueous solution to be less polar the emission intensity of TAMRA monomers in the hybrid D decreases. When CTAB, a cationic surfactant, was added to the solutions, the emission and absorption did not change until a concentration of $100 \mu \mathrm{M}$ $\mathrm{CTAB}$ was reached. At $100 \mu \mathrm{M}$ CTAB, the absorbance spectrum intensity increases, which implies that aggregation has formed in the solution (Fig. S1(a) and (b), ESI†). The positively charged CTAB molecules neutralize the negative charged phosphate backbone of the DNA strands, causing the DNA structures to aggregate and condense together with CTAB molecules due to their hydrophobicity. ${ }^{22,28}$ In this neutralized and aggregated state the fluorescence and absorbance properties of TAMRA dyes on the DNA are influenced not only by dimerization, but also by other quencher molecules such as the guanine bases. ${ }^{20,21}$ For instance, while emission quenching occurred as the monomer ratio was decreased in the control hybrid $\mathrm{D}$, the emission barely changed as the monomer ratio increased in the other control hybrids A, B and C (Fig. 2). Thus, it is assumed that the emission is influenced by not only the dimerization but also other quenchers such as guanine bases in DNA. However, when CTAB concentration was increased above $100 \mu \mathrm{M}$, the emission and the monomer ratios became similar to the ratio for the pristine structures (Fig. 2(b)). Since CTAB molecules form micelle structures at these higher concentrations, the negatively charged phosphate backbone in DNA becomes bound to the positively charged micelle surfaces, resulting in aggregated DNA structures dispersed on the CTAB micelles in the solution. As a result of redispersion of the aggregated DNA structures in the solution, the three TAMRA dye conjugated DNA structures become similar to the original configurations and show spectral properties like the pristine structures. In the third case, when SDS, an anionic surfactant, was added to the solutions, results were similar to what was observed for Triton X-100 (Fig. 2). The fluorescent emission and dimerization of TAMRA dye conjugated DNA structures (control A, B and C) were not affected due to repulsive forces between negatively charged DNA backbone and the anionic SDS molecules. However, when cations such as sodium $\left(\mathrm{Na}^{+}\right)$and magnesium $\left(\mathrm{Mg}^{2+}\right)$ were added to the solution, the 
fluorescence emission intensity of TAMRA dye conjugated DNA increased due to the reduction of dimerization. Apparently, the addition of cations forms a double layer which screens the repulsive forces between DNA backbone and SDS micelle, which now allows the TAMRA dyes to have hydrophobic interaction with interior of the micelle structures. As the TAMRA dyes become isolated in the micelle structure, dimerization is reduced and fluorescent emission is increased (Fig. 2). This emission enhancement was more clearly observed with divalent magnesium cation $\left(\mathrm{Mg}^{2+}\right)$ than with monovalent sodium cation $\left(\mathrm{Na}^{+}\right)$, which is the most certain due to more effective reduction in the DNA backbone with negative charge by the $\mathrm{Mg}^{2+}$ cations. With regard to the TAMRA conjugated hybrid $\mathrm{D}$, the decreasing solvent polarity resulting from increasing SDS concentration causes a reduction in emission intensity, which is similar to the results observed for Triton X-100.

\subsection{Enhanced FRET and antenna effect in ds-DNA structures}

In the initial case (pristine) without surfactants or metal ions, the three TAMRA dye conjugated DNA strands (I, II) when hybridized to the Texas Red conjugated strands (III, IV), the antenna effect values for the FRET ds-DNA structures E, F, G, and $\mathrm{H}$ were only $1.39,1.54,0.61$ and 1.42 , respectively (Fig. 3). These relatively low values, about 1 of the antenna effect, means that the fluorescent energy corresponding one donor is transferring to the acceptor in spite of the fact that the TAMRA donor to donor dyes (E \& G) and TAMRA donor to Texas Red acceptor dye (E, F \& H) on the FRET ds-DNA hybrid structures were well within favourable Förster distances $\left(1 / R^{6}\right.$, about $\left.1.2 \mathrm{~nm}\right)$, which should theoretically have a high FRET efficiency. As it was shown in the previous section, hydrophobic interactions between donor dyes (fluorophores) in close proximity causes dimerization and quenching that significantly reduces the fluorescent emission intensity. It is certainly likely that FRET dsDNA hybrids $\mathrm{E}$ and $\mathrm{G}$ have more severe dimerization, which would account for the low antenna effect value and inefficient FRET. This appears especially true for the FRET ds-DNA hybrid
G, with the lowest antenna effect 0.6, where all three TAMRA donors and the Texas Red acceptor are in close proximity (donors with 3 base spacing and donor to acceptor with equivalent of 1-2 base spacing). In the first case for using a surfactant for reducing dimerization and enhancing FRET in the ds-DNA hybrids E, F, G and H, the non-ionic surfactant Triton X-100 was tested at concentrations that ranged from $1 \mu \mathrm{M}$ to $10 \mathrm{mM}$. As shown in Fig. 3, the addition of Triton $\mathrm{X}$ alone did not enhance FRET or the antenna effect. Because Triton X-100 is an uncharged non-ionic polar surfactant, it is likely to have only minimal interaction with negative charged DNA backbone, as was discussed in the previous section. In the second case, where the cationic surfactant CTAB was used at concentrations of 100 $\mu \mathrm{M}$ and above, both the antenna effect value and fluorescent dye emissions increased, especially in FRET ds-DNA hybrid structures E and G (Fig. 3 and 4). However, the donor and acceptor intensity for the emission spectrum barely increased compared to the acceptor intensity for FRET ds-DNA hybrids in the SDS and cation environment (Fig. 3). It is hypothesized that DNA neutralization and aggregation in solution with $100 \mu \mathrm{M}$ or higher concentrations of CTAB cause not only a decrease of dimerization between the donor fluorophores through interaction with the micelles, but also further reduce emission quenching produced by other quenchers such as the guanine bases. In the third case, FRET ds-DNA hybrid structures in solutions with SDS concentrations ranging from $1 \mu \mathrm{M}$ to $1 \mathrm{mM}$ did not show any significant increase in their antenna effect values (Fig. 3). This is a strong indication that donor and acceptor dye dimerization was still occurring. However, when sodium $\left(\mathrm{Na}^{+}\right)$or magnesium $\left(\mathrm{Mg}^{2+}\right)$ cations were also present in the SDS solutions, the antenna effect values were significantly increased (Fig. 3), as were the fluorescent dye emission intensities (Fig. 4 and S3†). These increases were most pronounced for the FRET ds-DNA hybrid E and G structures, where the dimerization of the three TAMRA donor dyes in closer proximity (3 base spacing) was significantly reduced. As discussed in the previous section, it is hypothesized that screening effect of negatively charged DNA backbone by the cations $\left(\mathrm{Mg}^{2+}\right.$ in (a)

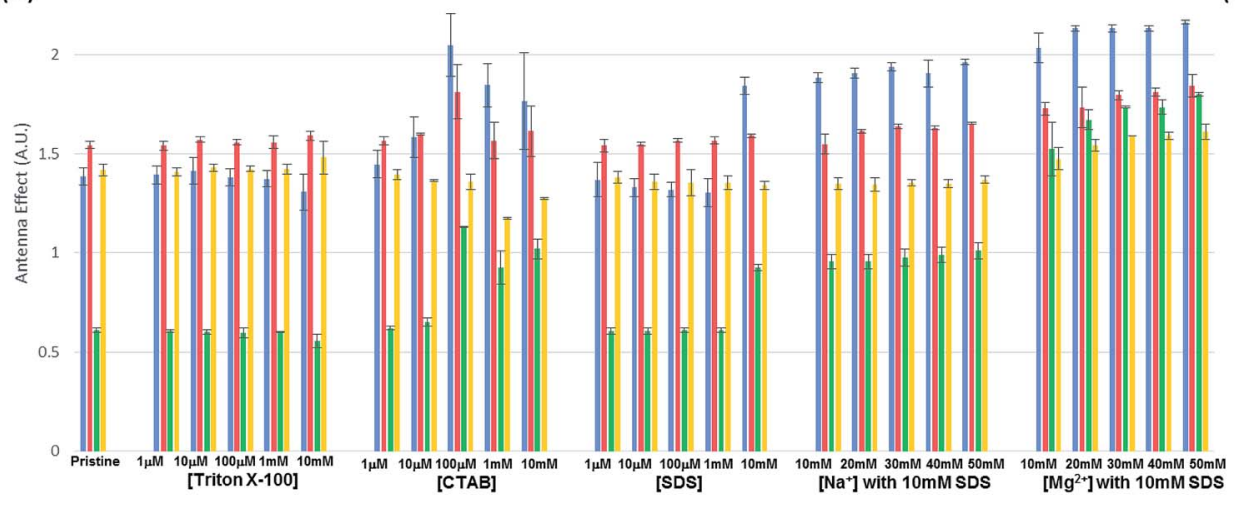

(b)

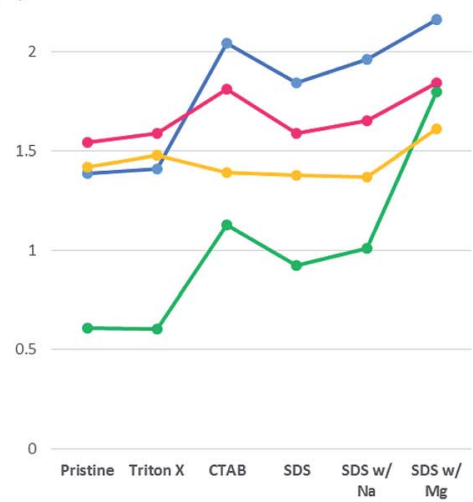

Fig. 3 Antenna effect values for FRET ds-DNA hybrid structures. (a) Antenna effect values for FRET ds-DNA hybrid structures E (Blue), F (Red), G (Green), and $\mathrm{H}$ (Yellow) with and without surfactants and metal ions; and (b) plot of the maximum FRET values for ds-DNA hybrid structures E (Blue), $F$ (Red), G (Green), and H (Yellow) in each surfactant/metal ion environment. 

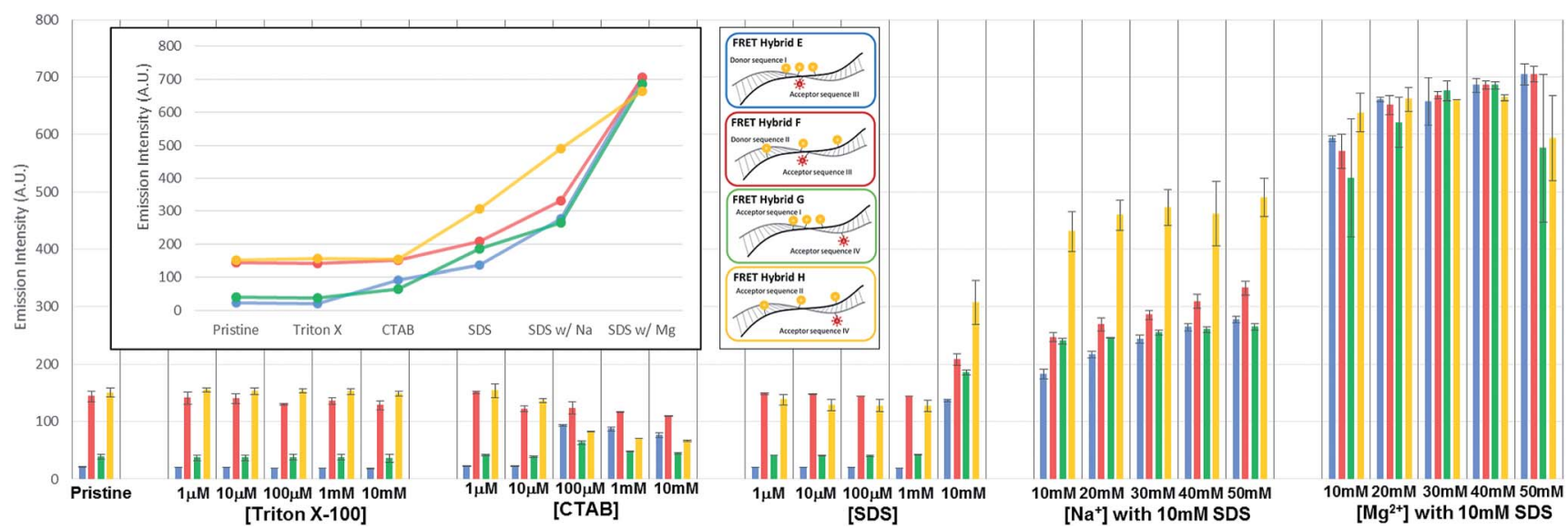

Fig. 4 Maximum intensity of Texas Red acceptor at $610 \mathrm{~nm}$ by decomposition (removal TAMRA emission overlap from Texas Red emission) for FRET hybrid E (Blue), F (Red), G (Green), and H (Yellow) when excited at $555 \mathrm{~nm}$ (inset: maximum fluorescent emission intensity for Texas Red acceptor under each surfactant/metal cation condition).

particular) allows the hydrophobic TAMRA dye fluorophores to form hydrophobic interaction with the SDS micelles that greatly reduces the dye dimerization and quenching, resulting in improved fluorescent dye emissions and quantum yield (Texas Red). In the best results case for reduced dimerization using SDS and $\mathrm{Mg}^{2+}$ cations, the overall antenna effect values where increased from 1.39 to 2.16 for $\mathrm{E}, 1.54$ to 1.84 for $\mathrm{F}, 0.61$ to 1.80 for $\mathrm{G}$ and 1.42 to 1.61 for $\mathrm{H}$ (see Fig. 3(b)). This is about a $15 \%$ increase for the $\mathrm{F}$ and $\mathrm{H}$ ds-DNA hybrid structures which had three TAMRA donors with 7 base spacing, where the Texas Red acceptor on the complementary strand was directly across from the center TAMRA in the F structure (equivalent to a 1-2 base spacing), and directly across from the $3^{\prime}$-terminal position TAMRA in the $\mathrm{H}$ structure (equivalent to a 1-2 base spacing). The antenna effect values increased more than $50 \%$ for the $\mathrm{E}$ ds-DNA structure with three TAMRA donors at 3 base spacing and the Texas Red acceptor directly across from the center TAMRA donor (equivalent to a 1-2 base spacing). Most interestingly, there was more than a $100 \%$ increase for the G dsDNA structure with three TAMRA donors at 3 base spacing and the Texas Red acceptor at a 3 base spacing across from the $3^{\prime}$-terminal TAMRA donor. In addition to the FRET ds-DNA structure $\mathrm{G}$ having benefited from the reduced dimerization and quenching effects by SDS surfactant and $\mathrm{Mg}^{2+}$ ion interactions, the relatively high final antenna effect value of 1.80 provides the most convincing evidence for extended FRET and the antenna effect. In the G ds-DNA structure, the two most distal TAMRA donors with a 7 base and 11 base spacing are beyond the Förster distance for efficient photonic transfer directly to the Texas Red acceptor. Finally, the best case FRET spectral information obtained in this study was also analyzed and presented in an alternate fashion. Fig. 5 again shows the fluorescence emission spectrum data for the Texas Red acceptor in FRET ds-DNA hybrids E, F, G, and $\mathrm{H}$ with and without $10 \mathrm{mM}$ SDS with $50 \mathrm{mM} \mathrm{Mg}^{2+}$ cation. The fluorescence emission spectrum of the Texas Red acceptor at $610 \mathrm{~nm}$ with excitation at

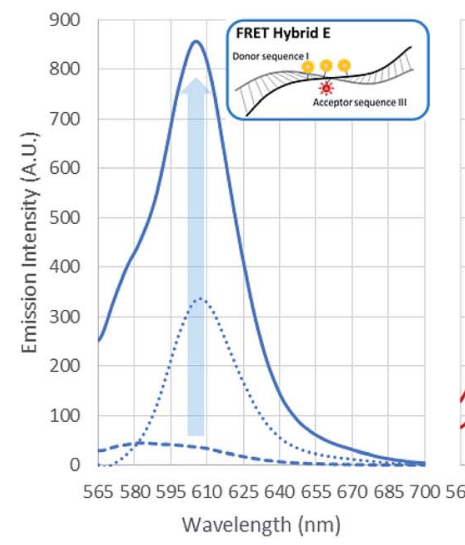

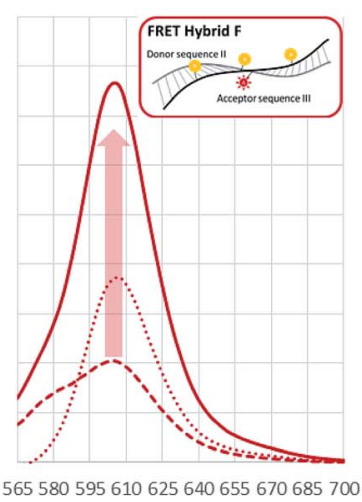

Wavelength $(\mathrm{nm})$

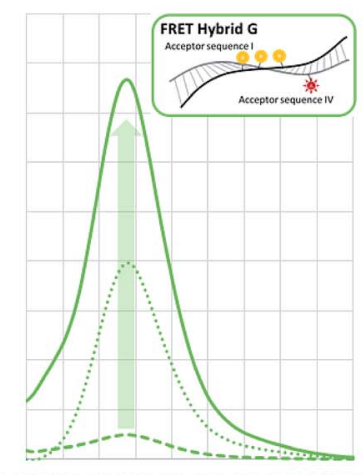

565580595610625640655670685 Wavelength $(\mathrm{nm})$

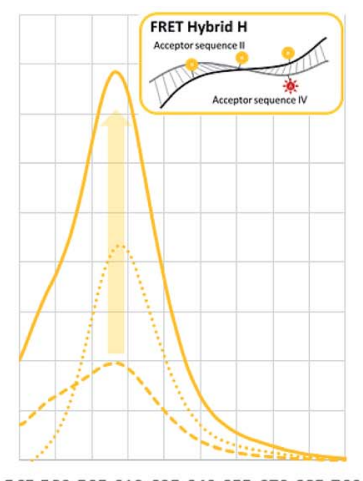

Wavelength $(\mathrm{nm})$

Fig. 5 Fluorescence emission spectrum of the Texas Red acceptor in FRET ds-DNA hybrids (E, F, G, H) with and without 10 mM SDS with 50 mM $\mathrm{Mg}^{2+}$ ion. Fluorescence emission spectrum of the Texas Red acceptor at $610 \mathrm{~nm}$ with excitation at $555 \mathrm{~nm}$ (Ex. max TAMRA) without SDS and $\mathrm{Mg}^{2+}$ ion (dash line). Fluorescence emission spectrum of the Texas Red acceptor at $610 \mathrm{~nm}$ with excitation at $595 \mathrm{~nm}$ (Ex. max Texas Red) with $10 \mathrm{mM} \mathrm{SDS}$ and $50 \mathrm{mM} \mathrm{Mg}^{2+}$ ion (dot line). Fluorescence emission spectrum of the Texas Red acceptor at $610 \mathrm{~nm}$ with excitation at $555 \mathrm{~nm}$ (Ex. max TAMRA) with $10 \mathrm{mM}$ SDS with $50 \mathrm{mM} \mathrm{Mg}^{2+}$ ion (solid line). 
$555 \mathrm{~nm}$ (Ex. max TAMRA) without SDS and $\mathrm{Mg}^{2+}$ cation is shown as the dashed line (- - ), the fluorescence emission spectrum of the Texas Red acceptor at $610 \mathrm{~nm}$ with excitation at $595 \mathrm{~nm}$ (Ex. max Texas Red) with $10 \mathrm{mM}$ SDS and $50 \mathrm{mM} \mathrm{Mg}^{2+}$ ion is shown as the dotted line $(\cdots)$, and the fluorescence emission spectrum of the Texas Red acceptor at $610 \mathrm{~nm}$ with excitation at $555 \mathrm{~nm}$ (Ex. max TAMRA) with $10 \mathrm{mM}$ SDS and $50 \mathrm{mM} \mathrm{Mg}^{2+}$ cation is shown as the solid color line (-). The results presented in this fashion again show the difference between the E and G ds-DNA structures and the $\mathrm{F}$ an $\mathrm{H}$ the ds-DNA structures, and overall help collaborate this study's conclusions.

\section{Conclusion}

Three surfactants Triton X-100, CTAB and SDS and two metal cations $\mathrm{Na}^{+}$and $\mathrm{Mg}^{2+}$ where investigated for their ability to reduce fluorescent quenching, improve FRET efficiencies and enhance the antenna effects in ds-DNA 21mer oligonucleotide constructs containing three fluorescent TAMRA donor dyes and one fluorescent Texas Red acceptor dye on a complementary 21mer oligonucleotide (Table 1 and Fig. 1). For the three TAMRA ss-DNA and ds-DNA structures with close inter-TAMRA distance (3 base spacing) hydrophobic based dimerizing interactions between the donor fluorophores lead to quenching and low fluorescence emissions. When Triton X-100, a neutral polar surfactant, was added to the solution at concentrations from 1 $\mu \mathrm{M}$ to $10 \mathrm{mM}$, the dimerization and the emission quenching of TAMRAs did not change, indicating no interaction with the DNA strands. However, when $100 \mu \mathrm{M}$ of $\mathrm{CTAB}$ was added in the solution, the cationic surfactant neutralizes the DNA backbone inducing aggregation which affects random behavior of the emission and dimerization of TAMRAs. In this aggregated form, it is assumed that the fluorescent emission is influenced by not only the dimerization but also other quenchers such as guanine bases in DNA. In the case of the SDS, an anionic surfactant, the surfactant itself did not reduce emission quenching due to repulsive forces between DNA and SDS micelles. However, if the concentration of cations such as sodium and magnesium, are increased in $10 \mathrm{mM}$ in the SDS solution, the dimerization of TAMRA dyes was significantly reduced and a very large increase in fluorescence emission was observed, especially in highly dimerized TAMRAs (strand IA and IB). It is hypothesized that the cations, particularly $\mathrm{Mg}^{2+}$ reduce the repulsive forces between the DNA backbone and the SDS micelle, allowing interaction and introduction of the fluorescent dyes into the SDS micelle by hydrophobic attraction. This appears to provide a sheathing effect that shields and/or insulates the TAMRA dyes conjugated on DNA, reducing dimerization and enhancing the fluorescent emission. When the three TAMRA donor sequences (I and II) and the Texas Red acceptor sequences (III and IV) were hybridized to form the FRET ds-DNA structures E, F, G and H, it was observed for all cases without the addition of surfactant and cations that dimerization of the fluorophores caused emission quenching leading to relatively low FRET efficiency and antenna effect values. Although non-ionic Triton X-100 did not influence the acceptor emission or the antenna effect, the antenna effect values did increase for the cationic CTAB surfactant at $100 \mu \mathrm{M}$ and higher concentrations. However, the emission intensity of the TAMRA donor and Texas Red acceptor barely increased, implying that the CTAB surfactant might not best suited to enhance FRET and the antenna effect in ds-DNA hybrid structures due to heavy aggregation induced by DNA neutralization. While the anionic SDS surfactant did not interact with the FRET ds-DNA hybrid structures due to repulsive electrostatic forces, the antenna effect values did increase significantly when divalent magnesium $\left(\mathrm{Mg}^{2+}\right)$ cation was also present in the $10 \mathrm{mM}$ SDS solution. Apparently, as SDS micelles could now approach the ds-DNA structures due to the charge screening effect of $\mathrm{Mg}^{2+}$ cations, the hydrophobic fluorophores were able to interact with the interior of the micelle, which reduces the dimerization and leads to an overall enhancement of fluorescent dye emissions, quantum yields and the antenna effect values $(2.16$ for $\mathrm{E}$, 1.84 for $\mathrm{F}, 1.80$ for $\mathrm{G}$ and 1.61 for $\mathrm{H}$ ). With regard to the FRET ds-DNA hybrid structure $\mathrm{G}$, which represents the best case for discriminating true long range FRET and antenna effects, the high antenna effect value of 1.80 provides convincing evidence that this was occurring. In the G ds-DNA structure, the two most distal TAMRA donors with 7 base and 11 base spacing are clearly beyond the Förster distance for transfer of photonic energy directly to the Texas Red acceptor. In order to account for the high antenna effect value of 1.80, the three TAMRA donors must be FRET coupled as an antenna that allows effective transfer of photonic energy captured by all three donors to be transferred by the proximal TAMRA donor (3 base spacing) to the Texas Red acceptor. Overall, this research study provides important insights and methods for not only using surfactants with cations to reduce fluorescent dye dimerization and quenching, but also for considering more optimal positioning of multiple fluorescent donor dye and acceptor dye molecules in unique ds-DNA structures designed with long range FRET and antenna properties. Such novel FRET ds-DNA structures would be useful for enabling a variety of more sensitive detection systems for research and clinical diagnostic applications, as well as for creating new programmable self-assembling 2D and 3D DNA based photonic nanostructures.

\section{Acknowledgements}

This work was supported in part by Award Number W81XWH14-2-0192 from the Defense Medical Research and Development Program and U.S. Army Medical Research Acquisition Activity (USAMRAA) and MJHeller Lab UCSD royalties from previous licensed technologies. The author would like to thank Taelin Oh and Joungmin Yoo who assisted in the graphic design of figures in the manuscript.

\section{References}

1 V. S. Jisha, K. T. Arun, M. Hariharan and D. Ramaiah, J. Phys. Chem. B, 2010, 114, 5912-5919.

2 L. Stryer, Annu. Rev. Biochem., 1978, 47, 819-846.

3 C.-Y. Zhang, H.-C. Yeh, M. T. Kuroki and T.-H. Wang, Nat. Mater., 2005, 4, 826-831. 
4 M. Heilemann, P. Tinnefeld, G. Sanchez Mosteiro, M. Garcia Parajo, N. F. Van Hulst and M. Sauer, J. Am. Chem. Soc., 2004, 126, 6514-6515.

5 R. Yan, D. Gargas and P. Yang, Nat. Photonics, 2009, 3, 569576.

6 S. J. Tan, M. J. Campolongo, D. Luo and W. Cheng, Nat. Nanotechnol., 2011, 6, 268-276.

7 M. J. Heller and L. E. Morrison, Rapid detection and identification of infectious agents, 1985, pp. 245-256.

8 R. A. Miller, A. D. Presley and M. B. Francis, J. Am. Chem. Soc., 2007, 129, 3104-3109.

9 M. J. Heller and R. H. Tullis, Nanotechnology, 1991, 2, 165.

10 I. Medintz and N. Hildebrandt, FRET-Förster Resonance Energy Transfer: From Theory to Applications, John Wiley \& Sons, 2013.

11 J. R. Unruh, G. Gokulrangan, G. Lushington, C. K. Johnson and G. S. Wilson, Biophys. J., 2005, 88, 3455-3465.

12 J. Kang, M. Loew, A. Arbuzova, I. Andreou and L. Dähne, Adv. Mater., 2010, 22, 3548-3552.

13 J. B. Randolph and A. S. Waggoner, Nucleic Acids Res., 1997, 25, 2923-2929.

14 Z. K. Majumdar, R. Hickerson, H. F. Noller and R. M. Clegg, J. Mol. Biol., 2005, 351, 1123-1145.

15 S. Doose, H. Neuweiler and M. Sauer, ChemPhysChem, 2009, 10, 1389-1398.

16 C. A. Seidel, A. Schulz and M. H. Sauer, J. Phys. Chem., 1996, 100, 5541-5553.
17 M. Torimura, S. Kurata, K. Yamada, T. Yokomaku, Y. Kamagata, T. Kanagawa and R. Kurane, Anal. Sci., 2001, 17, 155-160.

18 H. Kautsky, Trans. Faraday Soc., 1939, 35, 216-219.

19 H. Knibbe, D. Rehm and A. Weller, Ber. Bunsenges. Phys. Chem., 1968, 72, 257-263.

20 T. Heinlein, J.-P. Knemeyer, O. Piestert and M. Sauer, J. Phys. Chem. B, 2003, 107, 7957-7964.

21 S. Ranjit and M. Levitus, Photochem. Photobiol., 2012, 88, 782-791.

22 T. Oh, T. Takahashi, S. Kim and M. J. Heller, J. Biophotonics, 2016, 9, 49-54.

23 T. Oh, J.-Y. Choi and M. J. Heller, Analyst, 2016, 141, 23712375.

24 M. Ogawa, N. Kosaka, P. L. Choyke and H. Kobayashi, ACS Chem. Biol., 2009, 4, 535-546.

25 U. Rösch, S. Yao, R. Wortmann and F. Würthner, Angew. Chem., 2006, 118, 7184-7188.

26 M. A. Bahri, M. Hoebeke, A. Grammenos, L. Delanaye, N. Vandewalle and A. Seret, Colloids Surf., A, 2006, 290, 206-212.

27 M. Massey, W. R. Algar and U. J. Krull, Anal. Chim. Acta, 2006, 568, 181-189.

28 D. Santhiya and S. Maiti, J. Phys. Chem. B, 2010, 114, 76027608. 